\title{
NOUVELLE
}

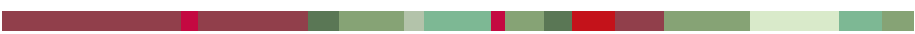

\section{Sténose du pylore et nNOS}

Dominique Labie, Ryad Tamouza

> La sténose hypertrophique du pylore est un accident relativement fréquent chez le nourrisson de race blanche (1,5/1000 naissances), à prédominance masculine nette $(4: 1)$. C'est la cause la plus fréquente de chirurgie au cours de la première année de vie. L'hypertrophie musculaire qui en est responsable ne se développe que dans les semaines qui suivent la naissance et les troubles sont très vite améliorés par une pyloromyotomie. Des études familiales ont, depuis longtemps, fait suspecter une composante génétique [1]. Ce spasme du pylore avec défaut de relaxation avait, par ailleurs, fait envisager un déficit en monoxyde d'azote (NO) dont le rôle comme médiateur de relaxation au niveau du tube digestif est connu. Les premiers arguments indirects en faveur de ce déficit remontent à 1992 [2]. L’hypothèse a été confirmée plus récemment par un premier travail effectué sur des biopsies chirurgicales prélevées chez six nourrissons et comparées à des prélèniveaux du tube digestif. De fait, les auteurs ont montré dans les prélèvements de sténose une diminution signifi-

cative du transcrit de la $\mathrm{NO}$-synthase neuronale (NOSI ou nNOS) [3].

À la même époque, les études de structure et de régulation de ce gène nNOS avaient plexe, l'expression, hétérogène en raison d'épissages alternatifs du transcrit, étant fonction des tissus et du stade de développement [4]. Un travail majeur, fruit d'une collaboration entre l'Université de Toronto (Canada) et I'Université Emory d'Atlanta (Georgia, USA), mettait en évidence la très grande diversité de traduction de la nNOS humaine [5]. Cette régulation, d'une complexité exceptionnelle, s'effectue à différents niveaux. Un premier niveau est l'usage alternatif de promoteurs multiples, ce qui affecte la localisation de l'initiation de transcription, et donc la séquence et la structure de I'ARNm. C'est ainsi que neuf variants d'un premier exon non traduit ont été mis en évidence, tous épissés au même exon 2, porteur du codon ATG initiateur de la traduction (Figure 1). La spécificité tissulaire est attestée par l'observation du groupement des variants en fonction des tissus où ils s'expriment; un groupe associe ceux qui sont exprimés dans les vements témoins effectués à différents montré sa régulation extrêmement com-

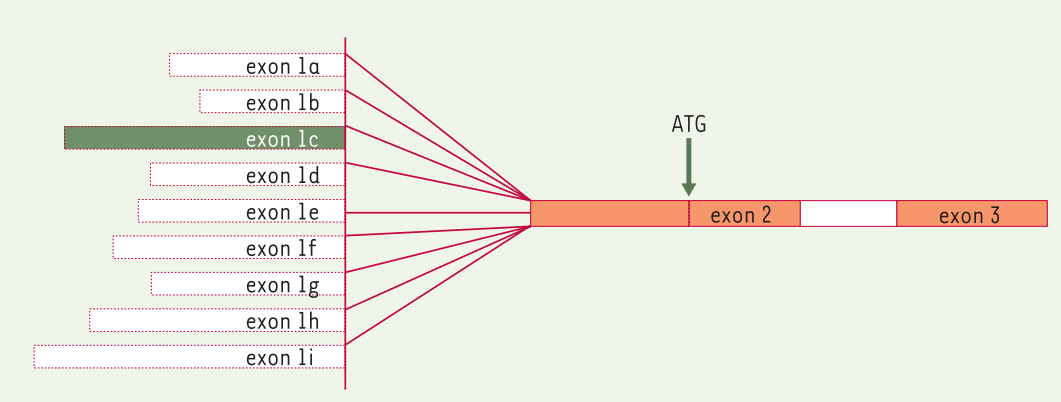

Figure 1. Gène de la NO-synthase. Représentation schématique des différents exons (neuf) pouvant être épissés au même exon 2. L'exon lc (vert) est le plus utilisé en situation normale et celui dont l'expression est effondrée dans certains cas de sténose hypertrophique du pylore.
D. Labie: Département de

Génétique, développement

et pathologie moléculaire,

Institut Cochin,

24, rue du Faubourg Saint

Jacques, 75014 Paris, France.

labie@cochin.inserm.fr

R. Tamouza: Laboratoire

d'Immunologie

et d'histocompatibilité,

Hôpital Saint Louis,

1, avenue Claude Vellefaux,

75010 Paris, France.

tamouza@

histo.chu-stlouis.fr

muscles squelettiques, l'autre ceux dont l'expression est enrichie dans les neurones. Un deuxième niveau de régulation se situe au niveau de la maturation de I'ARN. Deux anomalies d'épissage, au moins, ont été constatées: d'une part, l'existence d'un exon surnuméraire de 89 nt entre l'exon 1 non traduit et l'exon 2, et, d'autre part, une délétion fréquente dans un exon 2 de taille inhabituelle (1145nt), entre deux introns également très étendus. Cette dernière délétion, spécifique de tissu et du promoteur, devrait se traduire par des modifications de structure au niveau protéique. Un troisième niveau de variabilité, enfin, concerne la traduction, puisque les séquences leader non traduites varient entre 489 et 1033 nt. Ces longueurs, supérieures à la moyenne, et leur diversité, avaient fait supposer que l'extrémité 5' du transcrit pourrait intervenir de façon importante dans la régulation de la traduction, expliquant ainsi les différences observées dans les expériences de traduction in vitro avec un gène rapporteur luciférase. Une telle complexité de régulation de ce gène de 240 kb devait forcément retentir sur la fonction de la protéine: l'hypothèse avait été émise selon laquelle les inégalités de longueur de l'exon 1 devaient altérer des sites de fixation protéique, et sans doute retentir sur des voies de signalisation. La production d'une protéine à partir d'ARNm variables, 
qui ont utilisé divers promoteurs d'efficacité différente, devait aussi intervenir sûrement dans la physiopathologie et la réponse tissulaire.

Au cours d'un travail légèrement postérieur, en effet, une équipe allemande, de Munich, mettait en évidence la présence de variants d'épissage dans des prélèvements effectués à différents niveaux du tube digestif [6]. C'est ce même groupe de chercheurs qui a récemment poursuivi l'étude de l'expression de la NO-synthase neuronale dans la sténose hypertrophique du pylore chez le nourrisson et démontré le mécanisme moléculaire à l'origine de cette maladie [7]. Les auteurs ont d'abord identifié une réduction de l'ordre de $88 \%$ des transcrits de la NO-synthase dans des échantillons de pylore sténosé en comparaison avec des pylores normaux $(p<0,001)$. Le déficit quantitatif en $A R N m$, la diminution relative des neurones exprimant la nNOS qui s'ensuit, entraînent une hypertrophie et une hyperplasie des muscles lisses. L'étape suivante a été la détermination des promoteurs utilisés chez les patients et chez des témoins. Chez ces derniers, deux variants de l'exon 1 , lc et lf, ont des taux d'expression similaires, alors que dans les cas de sténose hypertrophique, l'effondrement des transcrits, s'il est global, porte majoritairement sur l'exon lc, variant prédominant en particulier dans les neurones. La séquence de cet exon lc a été entreprise, et comparée à celle de l'exon lc de 72 ADN témoins. Trois mutations différentes ont été identifiées chez trois patients différents. Mais, surtout, une mutation au niveau du promoteur $(-84 \mathrm{G} \rightarrow \mathrm{A})$ a été observée chez 10/16 patients ( 2 fois à l'état homozygote, 10 fois à l'état hétérozygote), alors qu'elle n'existe chez aucun témoin $(p=0,014)$.

Pour essayer de relier ce polymorphisme aux troubles fonctionnels, les auteurs sont revenus à une étude fonctionnelle de différents promoteurs de la nNOS qu'ils avaient faite antérieurement dans des lignées cellulaires [8]. L'exon lc quantitativement majoritaire avait été cloné et séquencé, le promoteur minimal, riche en GC et dénué de boîte TATA identifié. Les auteurs avaient, de plus, mis en évidence, en amont de ce promoteur minimal, entre - 90 et -47 , une zone paraissant importante par son extrême conservation dans l'évolution des espèces (homologie de $100 \%$ du rat à l'homme). La séquence nucléotidique de cette zone, appelée Staf, montrait son implication dans la fixation coopérative de plusieurs facteurs de transcription, membres de la famille Spl et ZNF (Figure 2). Différentes mutations de cette séquence consensus modifiaient le taux d'expression observé: abolition complète quand la boîte GC, fixant Spl, était mutée, réduction d'environ $53 \%$ par mutation du motif Staf. Or, c'est dans cette zone que les auteurs ont constaté, en cas de sténose du pylore,

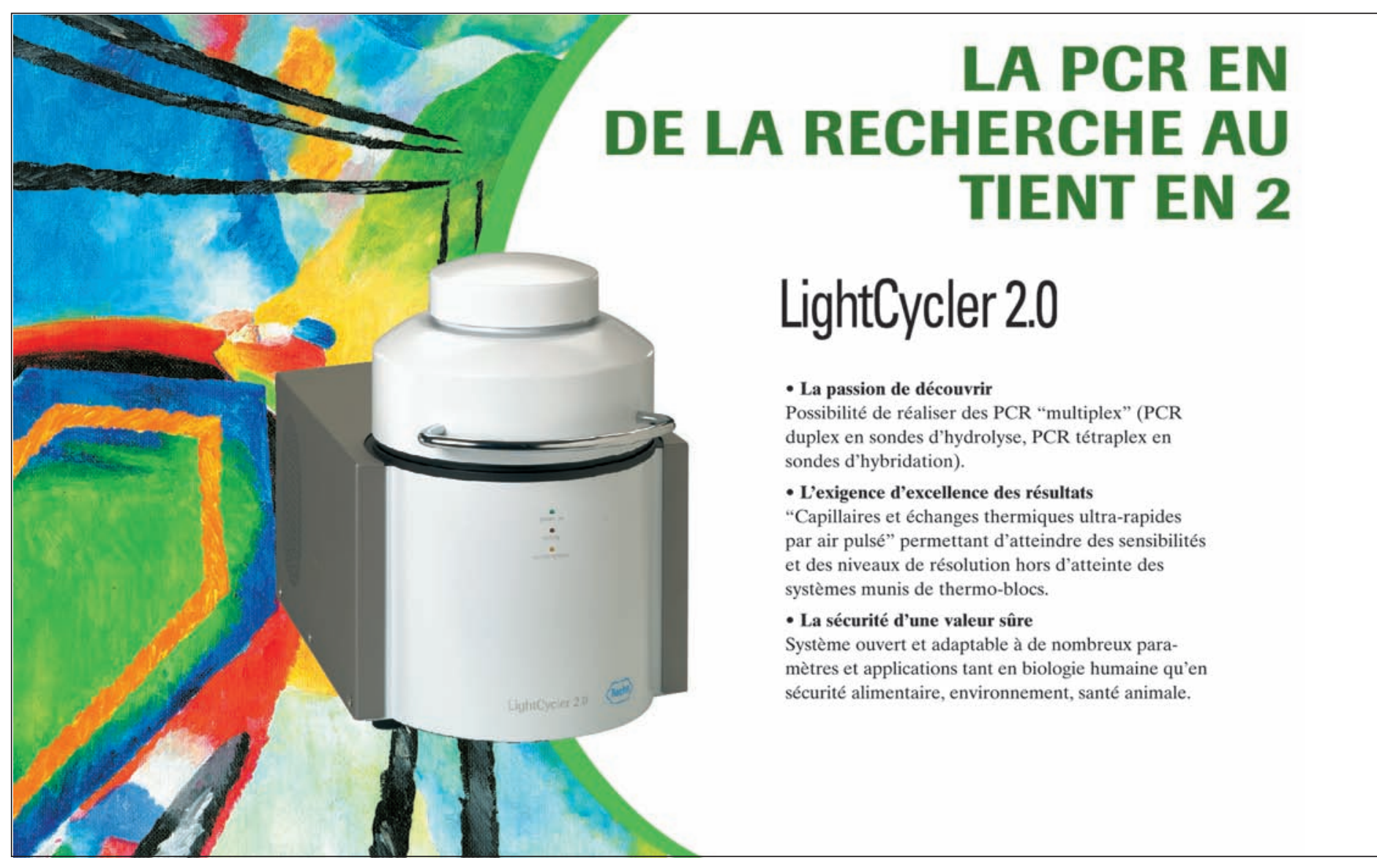




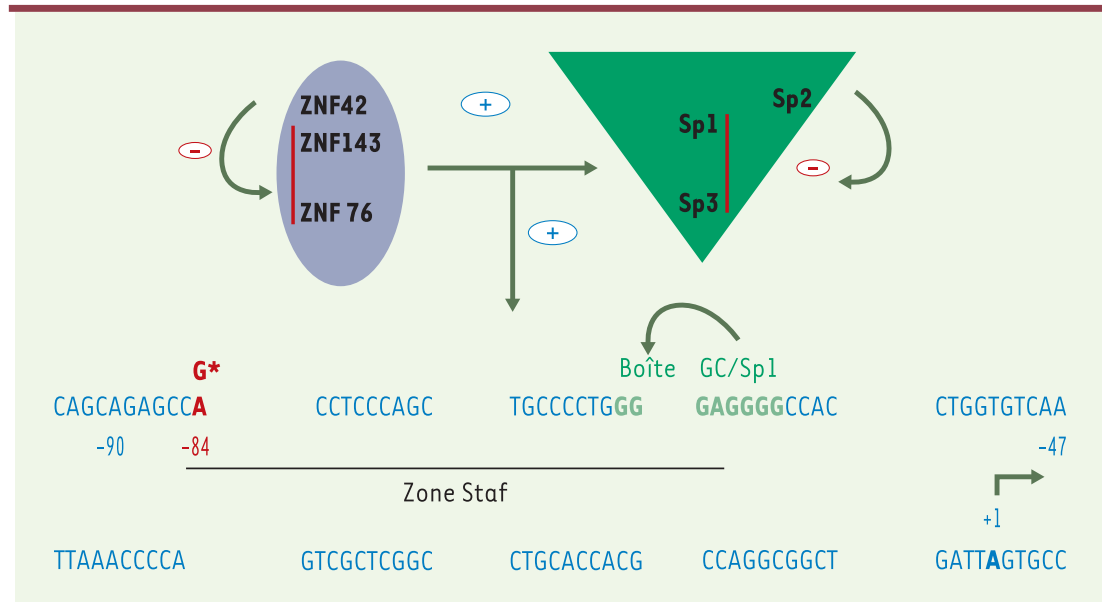

Figure 2. Interactions protéines-protéines au niveau du promoteur minimal du gène NOS-1. Le promoteur minimal du gène nNOS-1 (localisé entre -90 et 47 par rapport au site d'initiation de la transcription de l'exon lc) est porteur d'un site de fixation (boîte GC) pour les facteurs de transcription de la famille Spl. Parmi ceux-ci, seuls Spl, Sp3 et Sp4 sont capables d'interagir avec le site de fixation. Spl et sp3 ont un effet d'activation de la transcription additif, alors que Sp2 agit plutôt en réprimant l'effet de Spl. Le deuxième groupe de protéines activatrices est celui des facteurs ZNF: ZNF42, ZNF76, ZNF143. Alors que ZNF76 potentialise les effets activateurs de Spl et Sp3, ZNF143 présente un effet additif à celui de Spl, effet non observé avec Sp3. ZNF42 aurait un effet répresseur sur la transactivation, relayé par ZNF76, ZNF143 et Spl. La mutation trouvée en -84 interférerait avec ces interactions. le polymorphisme $-84 \mathrm{G} \rightarrow \mathrm{A}$. L'hypothèse physiopathologique, quoique dans l'incertitude de l'événement initial, est alors celle d'une réduction massive du transcrit prédominant (exon lc) qui, malgré une augmentation relative d'un exon If vicariant, entraîne une diminution globale de l'expression de nNOS et de la libération de NO. L'hypertrophie musculaire, le défaut de relaxation des sphincters, apparaissent comme des conséquences de ce déficit en NO, et, sans doute, de la cascade de signalisation NO/cGMP/cGMPkinase. Par l'utilisation d'un promoteur de substitution, le gène nNOS serait-il capable progressivement d'exprimer une quantité suffisante d'ARNm pour une évolution favorable? La survenue de troubles digestifs au cours de la première enfance, à une période où une nutrition satisfaisante est requise, rend actuellement l'option chirurgicale sûrement préférable. $\nabla$ Hypertrophic pyloric stenosis and neuronal nitric oxide synthase

\section{TEMPS RÉEL DIAGNOSTIC MÉDICAL SYSTÈMES}

\section{COBAS \\ TaqMan 48}

- $1^{\text {er }}$ et seul système de PCR en temps réel pour le diagnostic de routine.

- Système et réactifs marqués CE-IVD pour la quantification $\mathrm{HIV}, \mathrm{HCV}$ et $\mathrm{HBV}$.

- Performances inégalées en linéarité et seuils de sensibilité exceptionnels.

- Diagnostic PCR rendu en 2 à 3 heures.

\section{MARQUAGE CE-IVD}
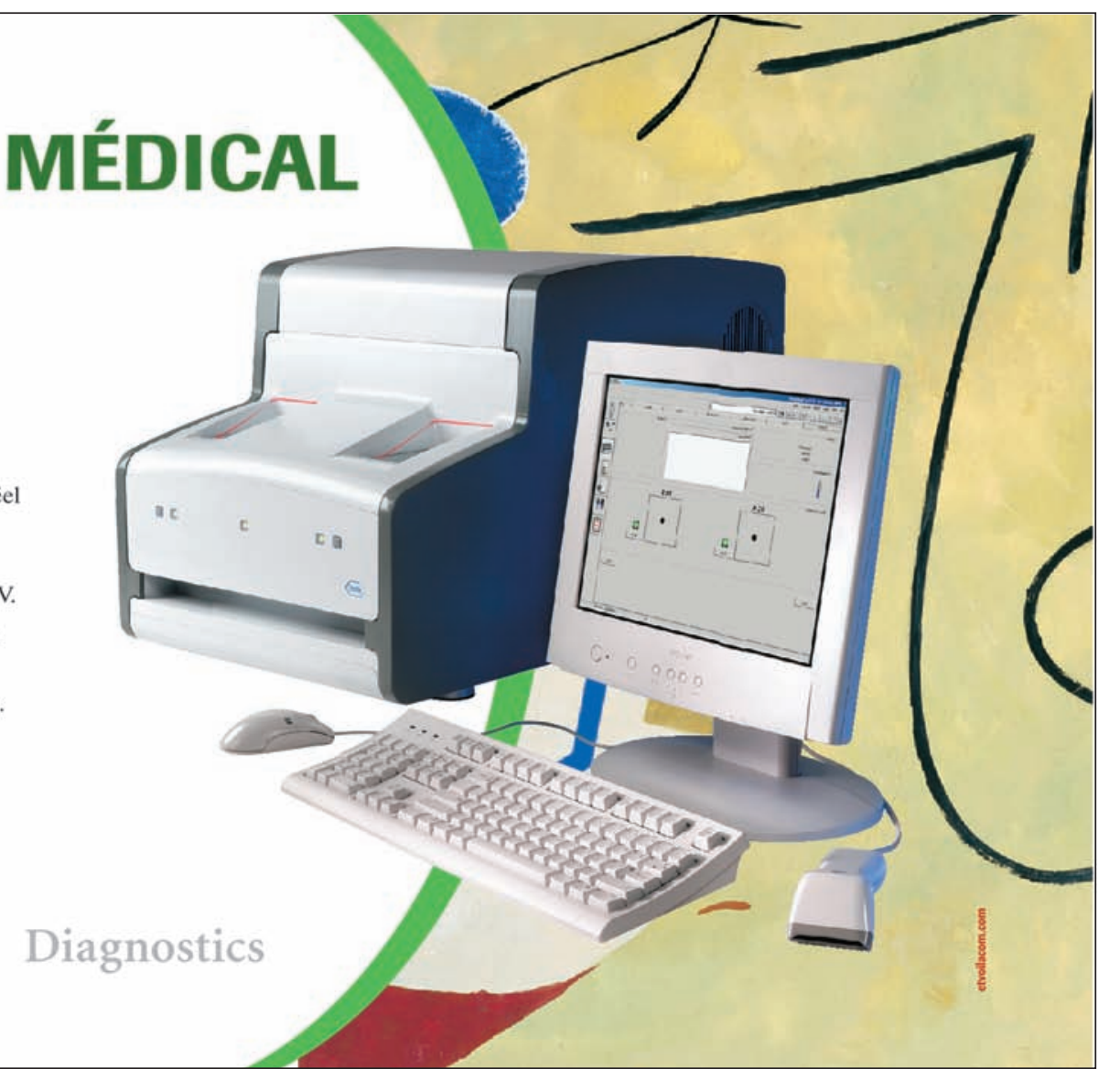


\section{RÉFÉRENCES}

1. Chung $\varepsilon$, Curtis $D$, Chen $G$, et al. Genetic evidence for the neuronal nitric oxide synthase gene (NOSl) as a susceptibility locus for infantile pyloric stenosis. Am J Hum Genet 1996; 58: 363-70.

2. Vanderwinden JM, Mailleux $P$, Schiffmann SN, et al. Nitric oxide synthase activity in infantile hypertrophic pyloric stenosis. N Engl J Med 1992; 327: 511-5.

3. Kusafuka T, Puri P. Altered messenger RNA expression of the neuronal nitric oxide synthase gene in infantile hypertrophic pyloric stenosis. Pediatr Surg Int 1997; 12: 576-9.
4. Lee MA, Cai L, Hubnner N, et al. Tissue- and development-specific expression of multiple alternatively spliced transcripts of rat neuronal nitric oxide synthase. J Clin Invest 1997;

100: 1507-12.

5. Wang $\mathrm{Y}$, Newton DC, Robb GB, et al. RNA diversity has profound effects on the translation of neurona nitric oxide synthase. Proc Natl Acad Sci USA 1999; 96: 12150-5

6. Saur D, Paehge H, Schusdziarra V, Allescher HD. Distinct expression of splice variants of neuronal nitric oxide synthase in the human gastrointestinal tract. Gastroenterology 2000; 118: 849-58.
7. Saur D, Vanderwinden JM, Seidler B, et al. Singlenucleotide promoter polymorphism alters transcription of neuronal nitric oxide synthase exon lc in infantile hypertrophic pyloric stenosis. Proc Natl Acad Sci USA 2004; 101 : 1662-7.

8. Saur D, Seidler B, Paehge H, et al. Complex regulation of human neuronal nitric-oxide synthase exon lc gene transcription. J Biol Chem 2002; 277: 25798-814.

\section{NOUVELLE}

\section{Pourquoi les limandes vivant dans I'Atlantique Nord ne gèlent pas?}

Christopher B. Marshall, Dominic Cuerrier, Nathalie Dourdin, Peter L. Davies

> Les poissons habitant les eaux polaires se sont adaptés à un environnement particulièrement hostile. Non seulement ils doivent maintenir une activité métabolique à des températures inférieures à zéro degré Celsius et contrôler leur osmolarité dans un environnement contenant près de $0,5 \mathrm{M}$ de sels, mais ils doivent aussi se protéger contre le gel. L'eau de mer, dont la concentration en sels est près de 2,5 fois plus élevée que celle du plasma sanguin, gèle à une température de $-1,9{ }^{\circ} \mathrm{C}$, température qu'elle atteint régulièrement pendant la saison hivernale. Puisque le plasma sanguin gèle à une température de $-0,8{ }^{\circ} \mathrm{C}$, les organismes inadaptés gèleraient rapidement dans cette eau contenant d'innombrables minuscules germes de cristaux de glace pouvant servir à tout moment comme centre de nucléation de la glace. Afin de se protéger contre ce phénomène, de nombreux poissons produisent des protéines antigel (PAG) [1], qui se fixent à la surface des cristaux de glace empêchant ainsi la croissance de ces cristaux (effet Kelvin). La présence de PAG abaisse le point de congélation d'une solution en dessous de son point de fusion colligatif et la différence, appelée «hystérésis thermale» (HT), est une propriété de la solution qui peut être mesurée en laboratoire.

Les poissons du nord de l'Atlantique, incluant les limandes (flets), les éperlans, les harengs, les lycodes, les chaboisseaux et les morues, fabriquent des PAG ainsi que des glycoprotéines antigel de cinq types différents, qui sont non homologues et remarquablement diverses en terme de séquence et de structure [1].

Les limandes plie rouge (Pleuronectes americanus) fabriquent une PAG

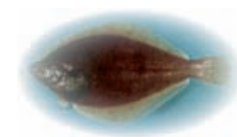
découverte il y a plus de 30 ans [2]. Cette PAG (de «type |») est composée d'une simple hélice $\alpha$ amphipatique de 33 acides aminés [3] qui se lie à la face pyramidale de la glace [4] via une surface riche en résidus alanine et complémentaire de celle du réseau cristallin de la glace. Cette protéine est fabriquée en grande quantité par le foie, d'où elle est exportée dans le sang à une concentration de $10-15 \mathrm{mg} / \mathrm{ml}$ [5], qui devrait résulter en une hystérésis thermale de $0,7{ }^{\circ} \mathrm{C}$ [6]. Cette activité antigel, lorsqu'elle est combinée à l'effet colligatif des solutés sanguins, pourrait contribuer à protéger les limandes jusqu'à une température de $-1,5^{\circ} \mathrm{C}$, mais les laisseraient vulnérables à la température de $-1,9^{\circ} \mathrm{C}$ des eaux du nord de l'Atlantique dans lesquelles elles vivent.

Afin de déterminer si la résistance des limandes au gel était due à des facteurs antigel supplémentaires, nous avons mesuré le degré de HT dans un échantillon de plasma soigneusement maintenu à basse température. Contre toute attente, le degré de HT était supérieur à $2{ }^{\circ} \mathrm{C}[7]$, soit plus de deux fois l'activité maximale de la PAG de «type |». De plus, les cristaux de glace présents dans le plasma étaient d'une forme fuselée complexe alors que les cristaux très particuliers dus à la présence de PAG de «type |» ont une forme de bipyramide hexagonale (Figure 1). Au contraire, lorsqu'un aliquot de l'échantillon était préalablement réchauffé à température ambiante, le degré de HT était largement réduit et les cristaux avaient alors une forme de bipyramide hexagonale. Ces 